\title{
SOME EXAMPLES RELATED TO KATO'S CONJECTURE
}

\author{
RHONDA J. HUGHES and PAUL R. CHERNOFF
}

(Received 13 April 1994; revised 21 September 1994)

Communicated by P. G. Dodds

\begin{abstract}
We show that the Kato conjecture is true for $m$-accretive operators with highly singular coefficients. For operators of the form $A=\tilde{D}^{*} F \tilde{D}$, where $\tilde{D}$ formally corresponds to $d / d x+z \delta$ on $L^{2}(\mathbb{R})$, we prove that $\operatorname{Dom}\left(A^{1 / 2}\right)=\operatorname{Dom}(\tilde{D})=e^{-z H} H^{1}(\mathbb{R})$, where $H$ is the Heavyside function. By adapting recent methods of Auscher and Tchamitchian, we characterize $\operatorname{Dom}(A)$ in terms of an unconditional wavelet basis for $L^{2}(\mathbb{R})$.
\end{abstract}

1991 Mathematics subject classification (Amer. Math. Soc.): 47B25, 47B44, $35 \mathrm{~J} 15$.

\section{Introduction}

Kato's conjecture, or the 'square root problem', involves the square root of certain elliptic operators that arise from sesquilinear forms defined on domains in $L^{2}\left(\mathbb{R}^{n}\right)$. To consider the simplest case, let $J$ be the sesquilinear form defined on $H^{1}(\mathbb{R})$ by

$$
J(f, g)=\int F(x) f^{\prime}(x) \overline{g^{\prime}(x)} d x,
$$

where $F \in L^{\infty}(\mathbb{R})$ and $\operatorname{Re} F(x) \geq \gamma>0$ for some constant $\gamma$. Then $J$ determines a maximal accretive operator $T$ (which is formally $(-d / d x) F(d / d x)$ ). It is wellknown that $T$ has a maximal accretive square root, defined by

$$
T^{1 / 2} f=\frac{2}{\pi} \int_{0}^{\infty}\left(I+t^{2} T\right)^{-1} T f d t
$$

(cf. [8]); the conjecture of Kato is that the domain of $T^{1 / 2}$ is $H^{1}(\mathbb{R})$. In one dimension this was first proved by Coifman, McIntosh and Meyer (cf. [5, 6]). More recently,

Research supported in part by a grant from the National Science Foundation.

(C) 1996 Australian Mathematical Society 0263-6115/95 \$A2.00+0.00 
Auscher and Tchamitchian gave a new, simple proof (cf. [1, 2]), using a wavelet construction due to Tchamitchian ([16]). If $n \geq 2$, only partial results have been obtained (by Fabes, Jerison, and Kenig [7]); in general, the question remains open. In this paper we adapt the method of Auscher and Tchamitchian to a family of operators with highly singular coefficients that is based on examples introduced in [4], and show that the domains of their square roots satisfy the Kato conjecture.

The Kato conjecture is related to the subtle issue of the representation of forms by square roots (cf. [10]). If the form $J$ is closed, symmetric, and bounded below, then there is an associated self-adjoint operator $T$ with the property that $\operatorname{Dom}\left(T^{1 / 2}\right)=$ $\operatorname{Dom}(J)$, and

$$
J(f, g)=\left(T^{1 / 2} f, T^{1 / 2} g\right),
$$

for all $f, g \in \operatorname{Dom}(J)$. (This is the 'second representation theorem' in [8, p. 331]). The situation is somewhat different for non-symmetric forms. In fact, McIntosh showed that in general, $\operatorname{Dom}\left(T^{1 / 2}\right)$ and $\operatorname{Dom}\left(T^{* 1 / 2}\right)$ need not coincide; in [10], he provides conditions under which a form $J$ is representable by square roots in the sense that

$$
\operatorname{Dom}\left(T^{1 / 2}\right)=\operatorname{Dom}\left(T^{* 1 / 2}\right)=\operatorname{Dom}(J),
$$

and

$$
J(f, g)=\left(T^{1 / 2} f, T^{* 1 / 2} g\right) .
$$

Moreover, he relates this difficulty to deep results of Calderón on norm inequalities for commutators of pseudodifferential operators, as well as to subtle multilinear estimates for singular integral operators. This relationship is central to the proof of the Kato conjecture in [5], and is thoroughly discussed in the survey article by McIntosh [12].

Viewed in another way, the Kato conjecture is connected to problems in perturbation theory. It is well-known that if $H_{0}$ is a semi-bounded self-adjoint operator, and $V$ is a symmetric form that is small with respect to $H_{0}$ in the sense that $\operatorname{Dom}(V) \supseteq Q\left(H_{0}\right)$, (where $Q(H)$ is the form domain of the operator $H$, cf. [14, p. 167]) and

$$
|V(\phi, \phi)| \leq a\left(H_{0} \phi, \phi\right)+b\|\phi\|^{2}
$$

for constants $0 \leq a<1, b \in \mathbb{R}$, and all $\phi \in \operatorname{Dom}\left(H_{0}\right)$, then the form sum $H_{0}+V$ is a semi-bounded symmetric form, and so corresponds to a self-adjoint operator $H$ (formally $H_{0}+V$ ) with $Q(H)=Q\left(H_{0}\right)$. Note that it follows from the spectral theorem that $Q(H)=\operatorname{Dom}\left(|H|^{1 / 2}\right)$. If $H_{0}$ is not bounded below, the result is no longer true, even for symmetric forms (cf. [8, p. 341]). This result can be related to the form domain question for square roots by considering the form $\mathrm{H}-\mathrm{H}_{0}$, where $\mathrm{H}_{0}$ is a self-adjoint operator, and $H$ is a symmetric form with domain $Q\left(H_{0}\right)$. If $H-H_{0}$ is small with respect to $\left|H_{0}\right|$, then $H$ determines a self-adjoint operator (the so-called pseudo-Friedrichs extension, cf. [8, p. 341]), and the relevant question is whether $Q(H)=\operatorname{Dom}\left(|H|^{1 / 2}\right)=Q\left(H_{0}\right)$. In general, the answer is no (cf. [10]). 
In a formal sense, the operator $T$ determined by the form $\left(F f^{\prime}, g^{\prime}\right)$ on $H^{1}$ may be viewed as a singular perturbation of $H_{0}=-F\left(d^{2} / d x^{2}\right)$. Although there is no form corresponding to $H_{0}$, it is shown in [13] for certain $F$, (and in [9] in general) that $\operatorname{Dom}\left(H_{0}{ }^{1 / 2}\right)=H^{1}$. On the other hand, since the Kato conjecture is true, $\operatorname{Dom}\left(T^{1 / 2}\right)=$ $H^{1}$. That is, the domain of the square root of the 'perturbed operator' coincides with the domain of the square root of the 'unperturbed' operator. We will see that for the operators considered here, the Kato conjecture is true, whereas the domain of the square root of the perturbed operator does not coincide with the domain of the square root of the unperturbed operator. In a sense, perturbation theory and the theory of quadratic forms provide different answers for the square root question as it pertains to the examples described in the next section.

\section{Generalized elliptic operators}

Recently, Auscher and Tchamitchian proved Kato's conjecture for second order elliptic operators on an open set in $\mathbb{R}$, with arbitrary boundary conditions, thereby extending the results in [5]. Their proof relies on the reduction of the general case to the (formal) operator $(-d / d x) F(d / d x)$, whose domain is spanned by an unconditional (Riesz) basis of wavelets adapted to the function $F(x)$. Here, we extend these results to a family of operators based on examples in [4], where we introduce a new class of point interactions that are explicitly solvable in terms of their spectral and scattering properties. These operators arise from sesquilinear forms, and involve more singular coefficients than those in [2]; formally, they have the form $A=\tilde{D}^{*} F \tilde{D}$, where $\tilde{D}=d / d x+z \delta$, in the sense defined by Segal (cf. [15]). We will show in Sections 35 that the Kato conjecture is true for these operators; that is, $\operatorname{Dom}\left(A^{1 / 2}\right)=\operatorname{Dom}(\tilde{D})=$ $e^{-z H} H^{1}(\mathbb{R})$, where $H$ is the Heaviside function. We proceed by reducing the problem to the case of the second-order term, as in [2], and by modifying the wavelet basis for $L^{2}(\mathbb{R})$ used in [1]. We will see, however, that as formal perturbations of $F(x)\left(d^{2} / d x^{2}\right)$, these examples are too singular to preserve form domains in the sense described at the end of the last section.

For $r \in \mathbb{R}, z \in \mathbb{C}$, define

$$
Q_{\{r, z\}}=\left(r+T_{z}\right)^{*}\left(r+T_{z}\right)
$$

where $T_{z}=e^{-2 H}(d / d x) e^{2 H}, H$ is the Heaviside function, and

$$
\operatorname{Dom}\left(T_{z}\right)=\left\{\phi \in L^{2}(\mathbb{R}) \mid e^{z H} \phi \in H^{1}(\mathbb{R})\right\} ;
$$

that is, $\phi \in H^{1}(\mathbb{R} \backslash\{0\})$, and $\phi\left(0^{+}\right)=e^{-z} \phi\left(0^{-}\right)$. Although $T_{z}$ is formally $d / d x+z \delta$, it is argued in [4] that a more reasonable interpretation involves a renormalization of the 
coupling constant, so that $z$ is replaced by $2 \tanh (z / 2)$. Continuing the development, define

$$
L_{\{r, z\}}=Q_{\{r, z\}}-r^{2} I,
$$

where $I$ is the indentity operator on $L^{2}(\mathbb{R})$. It is easy to see that $\operatorname{Dom}\left(L_{\{r, z\}}\right)$ is prescribed by the two boundary conditions:

$$
\begin{aligned}
\phi\left(0^{+}\right) & =e^{-z} \phi\left(0^{-}\right), \\
r \phi\left(0^{+}\right)+\phi^{\prime}\left(0^{+}\right) & =e^{\bar{z}}\left[r \phi\left(0^{-}\right)+\phi^{\prime}\left(0^{-}\right)\right] .
\end{aligned}
$$

$L_{\{r, z\}}$ is a self-adjoint extension of $-\Delta \mid C_{0}^{\infty}(\mathbb{R} \backslash\{0\})$ and, when expanded formally, corresponds to a perturbation of the Laplacian by pseudopotentials (which are not rigorously defined) involving $\delta$ and $\delta^{2}$ terms. We show in [4] that these operators can be approximated in the strong resolvent sense by operators with local short range potentials.

Recall now that if $H_{0}$ is a a self-adjoint operator acting on a Hilbert space $\mathscr{H}$, then the form domain $Q\left(H_{0}\right)$ is defined by

$$
Q\left(H_{0}\right)=\left\{\phi \in \mathscr{H}\left|\int\right| \lambda \mid d(E(\lambda) \phi, \phi)<\infty\right\},
$$

where $H_{0}=\int \lambda d E(\lambda)$ is the spectral representation of $H_{0}$. Also, $Q\left(H_{0}\right)=$ $\operatorname{Dom}\left(\sqrt{\left|H_{0}\right|}\right)$; by the spectral theorem $\sqrt{\left|H_{0}\right|}=\int|\lambda|^{1 / 2} d E(\lambda)$.

PROPOSITION 1. $Q\left(L_{\{r, z\}}\right)=e^{-z H} H^{1}(\mathbb{R})$.

PROOF. Since bounded perturbations preserve form domains,

$$
Q\left(L_{\{r, z\}}\right)=Q\left(\left(T_{z}+r I\right)^{*}\left(T_{z}+r I\right)\right)=\operatorname{Dom}\left(\sqrt{\left(T_{2}+r I\right)^{*}\left(T_{z}+r I\right)}\right) .
$$

But $\sqrt{A^{*} A}=|A|$, (cf. $[\mathrm{K}]$ ), so that the latter is equal to

$$
\operatorname{Dom}\left(\left|T_{z}+r I\right|\right)=\operatorname{Dom}\left(T_{z}+r I\right)=\operatorname{Dom}\left(T_{z}\right)=e^{-z H} H^{1}(\mathbb{R}) .
$$

We see immediately from the perturbation theory viewpoint that unless $z=0$, $Q\left(L_{\{r, z\}}\right) \neq Q(-\Delta)$, despite the fact that these operators are formally perturbations of $-\Delta$ by quadratic forms, and are explicitly strong resolvent limits of operators whose form domains are $H^{1}(\mathbb{R})$. On the other hand, these operators arise from the forms

$$
J_{\{r, z\}}(\phi, \psi)=\left(\left(T_{z}+r I\right) \phi,\left(T_{z}+r I\right) \psi\right)
$$


with domain $e^{-z H} H^{1}(\mathbb{R})$, and as such support the Kato conjecture. Indeed, $J_{\{r, z\}}$ is a closed, densely-defined positive form with associated self-adjoint operator $L_{\{r, z\}}+r^{2} I$, and so

$$
\operatorname{Dom}\left(J_{\{r, z\}}\right)=e^{-z H} H^{1}(\mathbb{R})=\operatorname{Dom}\left(\sqrt{L_{\{r, z\}}+r^{2} I}\right)=\operatorname{Dom}\left(\sqrt{L_{\{r, z\}}}\right)=Q\left(L_{\{r, z\}}\right) .
$$

As observed by Chernoff, we can expand this family to include $-\Delta+c \delta$ by considering the extended family of operators

$$
L_{\{r, s, z\}}=\left(T_{z}+r H(x)+s H(-x)\right)^{*}\left(T_{z}+r H(x)+s H(-x)\right)-r^{2} H(x)-s^{2} H(-x)
$$

where $z \in \mathbb{C}, r, s \in \mathbb{R}$. Then $L_{\{r, s, z\}}$ is a self-adjoint extension of $-\Delta \mid C_{0}^{\infty}(\mathbb{R} \backslash\{0\})$ and its domain corresponds to the conditions $\phi \in \operatorname{Dom}\left(T_{z}\right)$ and $\left(T_{z}+r H(x)+s H(-x)\right) \phi \in$ $\operatorname{Dom}\left(T_{z}^{*}\right)$. That is,

$$
\begin{aligned}
\phi\left(0^{+}\right) & =e^{-z} \phi\left(0^{-}\right), \\
r \phi\left(0^{+}\right)+\phi^{\prime}\left(0^{+}\right) & =e^{\bar{z}}\left[s \phi\left(0^{-}\right)+\phi^{\prime}\left(0^{-}\right)\right] .
\end{aligned}
$$

In the notation of [4], this corresponds to the boundary conditions

$$
\begin{aligned}
\phi\left(0^{+}\right) & =\alpha \phi\left(0^{-}\right)+\beta \phi^{\prime}\left(0^{-}\right), \\
\phi^{\prime}\left(0^{+}\right) & =\gamma \phi\left(0^{-}\right)+\eta \phi^{\prime}\left(0^{-}\right)
\end{aligned}
$$

with $\alpha=e^{-z}, \beta=0, \gamma=e^{\bar{z}} s-e^{-z} r, \eta=e^{\bar{z}}$. Note that when $z=0$, the boundary conditions become

$$
\begin{aligned}
\phi\left(0^{+}\right) & =\phi\left(0^{-}\right), \\
\phi^{\prime}\left(0^{+}\right)-\phi^{\prime}\left(0^{-}\right) & =(s-r) \phi(0),
\end{aligned}
$$

which are the boundary conditions for $-\Delta+c \delta$, with $c=s-r$.

It follows from essentially the same argument as that given above that $\operatorname{Dom}\left(L_{\{r, s, z)}^{1 / 2}\right)$ $=e^{-z H} H^{1}(\mathbb{R})$, so that when $z=0$, we have the familiar result that $Q(-\Delta+c \delta)=$ $H^{1}(\mathbb{R})$.

Continuing our development, let $F \in L^{\infty}(\mathbb{R})$ with $\operatorname{Re} F(x) \geq \rho>0$ for some constant $\rho$. Then $J(f, g)=\left(F f^{\prime}, g^{\prime}\right)$, defined on $H^{1}(\mathbb{R})$, is a closed sectorial form (cf. $[8,6])$ that gives rise to an $m$-accretive operator $T$ which is formally $(-d / d x) F(d / d x)$. In [2], this result is extended to the case where $J$ is replaced by the sesquilinear form

$$
J(f, g)=\left(a f^{\prime}, g^{\prime}\right)+\left(b f, g^{\prime}\right)+\left(c f^{\prime}, g\right)+(d f, g),
$$

on $\operatorname{Dom}(V) \subseteq H^{1}(\mathbb{R})$, and $a, b, c, d \in L^{\infty}(\mathbb{R})$, with $\operatorname{Re} a(x) \geq 1$ or, more generally, to $\operatorname{Dom}(V) \subseteq H^{1}(\Omega)$, where $\Omega$ is an open set in $\mathbb{R}$. 
We now consider the family of sesquilinear forms

$$
J_{\{r, s, z\}}(f, g)=\left(\left(F\left(T_{z}+r H(x)+s H(-x)\right) f,\left(T_{z}+r H(x)+s H(-x)\right) g\right)\right.
$$

with domain $e^{-z H} H^{1}(\mathbb{R})$, thereby introducing more singular coefficients than in (1). Note that when $z=0$, these forms are a special case of those in [2]. However, when $z \neq 0$, the coefficients are considerably more singular than those studied there. Observe further that if $F \equiv 1$ (and $z=0$ ), the corresponding (self-adjoint) operator is $-\Delta+(s-r) \delta+\left(r^{2}+s^{2}\right) I$.

PROPOSITION 2. For $z \in \mathbb{C}, r, s \in \mathbb{R}, J_{\{r, s, z]}$ is a closed sectorial form with domain $e^{-z H} H^{1}(\mathbb{R})$.

PROOF. It is well known that the form $J$ in (1) above is a closed sectorial form on $H^{1}(\mathbb{R})$ (cf. $[\mathrm{K}]$ ), and that for suitable $\gamma \in \mathbb{R}, 0 \leq \theta<\pi / 2$,

$$
\operatorname{Re}(J(\phi, \phi))+\gamma\|\phi\|^{2} \geq 0
$$

and

$$
|\operatorname{Im} J(\phi, \phi)| \leq \tan \theta\left(\operatorname{Re} J(\phi, \phi)+\gamma\|\phi\|^{2}\right) .
$$

Then for $f \in e^{-z H} H^{1}(\mathbb{R}), f=e^{-z H} \phi$ for $\phi \in H^{1}(\mathbb{R})$, and $J_{\{r, s, z\}}(f, f)=J(\phi, \phi)$, where

$$
\begin{aligned}
a(x) & =e^{-2 \operatorname{Re}(z) H} F(x), \\
b(x) & =e^{-2 \operatorname{Re}(z) H} F(x)[r H(x)+s H(-x)], \\
c(x) & =e^{-2 \operatorname{Re}(z) H} F(x)[r H(x)+s H(-x)], \\
d(x) & =e^{-2 \operatorname{Re}(z) H} F(x)\left[r^{2} H(x)+s^{2} H(-x)\right] .
\end{aligned}
$$

The functions $a(x), b(x), c(x)$, and $d(x)$ are in $L^{\infty}(\mathbb{R})$ and $\operatorname{Re}(a(x)) \geq e^{-2 \operatorname{Re}(z)} \rho=$ $\tilde{\rho}>0$. Clearly,

$$
\operatorname{Re}\left(J_{\{r, s, z\}}(f, f)\right)=\operatorname{Re}(J(\phi, \phi)) \geq-\gamma\|\phi\|^{2} \geq-\tilde{\gamma}\|f\|^{2},
$$

and

$$
\begin{aligned}
\left|\operatorname{Im} J_{\{r, s, z]}(f, f)\right| & =|\operatorname{Im} J(\phi, \phi)| \leq \tan \theta\left(\operatorname{Re} J(\phi, \phi)+\gamma\|\phi\|^{2}\right) \\
& \leq \tan \theta\left(\operatorname{Re}\left(J_{\{r, s, z]}(f, f)\right)+\tilde{\gamma}\|f\|^{2}\right)
\end{aligned}
$$

so that $J_{\{r, s, 2\}}$ is sectorial; the form is closed because $J$ is closed. 
We remark that Proposition 2 also follows from [6, Theorem 10.3].

By the first representation theorem for sesquilinear forms (cf. [8, Theorem VI.2.1]), there exists an $m$-sectorial operator $A_{\{r, s, z\}}$ such that $\operatorname{Dom}\left(A_{\{r, s, z\}}\right) \subseteq e^{-z H} H^{1}(\mathbb{R})$, and $J_{\{r, s, z\}}(f, g)=\left(A_{\{r, s, z\}} f, g\right)$, for all $f \in \operatorname{Dom}\left(A_{\{r, s, z\}}\right), g \in e^{-z H} H^{1}(\mathbb{R})$. In particular, for some $\nu \in \mathbb{R}, A_{\{r, s, z\}}+\nu$ is $m$-accretive, and so has an $m$-accretive square root. We then have

PROPOSITION 3. For $r, s, \gamma \in \mathbb{R}$,

$$
\operatorname{Dom}\left(\left(A_{\{r, s, i \gamma\}}+v\right)^{1 / 2}\right)=e^{-i \gamma H} H^{1}(\mathbb{R}) .
$$

PROOF. For simplicity, we assume that $v=0$. Following the ideas in [2], we begin by reducing the problem to the case of the second-order term only. In [2, Proposition 1], this reduction is done for the form $J$ in (1); namely, if

$$
\operatorname{Dom}\left(\left(-\frac{d}{d x} F \frac{d}{d x}\right)^{1 / 2}\right)=H^{1}(\mathbb{R})
$$

for all accretive $F \in L^{\infty}$, then the same is true for the domain of the square root of the $m$-accretive operator corresponding to the form $J$ (which contains lower order terms).

To see that this reduction is also possible for the form $J_{\{r, s, z\}}$, we introduce the notation $\tilde{D}=e^{-z H}(d / d x) e^{z H}$, with $\operatorname{Dom}(\tilde{D})=e^{-z H} H^{1}(\mathbb{R})$, and consider the (closed) form:

$$
\tilde{J}(f, g)=(F \tilde{D} f, \tilde{D} g), \quad f, g \in e^{-\imath H} H^{1}(\mathbb{R})
$$

As was shown above, $\tilde{J}$ is sectorial and determines an $m$-accretive operator $\tilde{A}$. Suppose that all such operators satisfy $\operatorname{Dom}\left(\tilde{A}^{1 / 2}\right)=e^{-z H} H^{1}(\mathbb{R})$. Following the proof of [2, Proposition 1] verbatim, set

$$
\tilde{T}=\tilde{D}^{*} a \tilde{D}+\tilde{D}^{*} b+c \tilde{D}+d, \quad \tilde{T}_{1}=\tilde{D}^{*} a \tilde{D}+\tilde{D}^{*} b, \quad \tilde{T}_{2}^{*}=\tilde{D}^{*} \bar{a} \tilde{D},
$$

where we note that $\tilde{D}^{*}=e^{\bar{z} H}(d / d x) e^{-\bar{z} H}$, and $a, b, c$, and $d$ are given by

$$
\begin{aligned}
& a(x)=F(x), \\
& b(x)=F(x)[r H(x)+s H(-x)], \\
& c(x)=F(x)[r H(x)+s H(-x)], \\
& d(x)=F(x)\left[r^{2} H(x)+s^{2} H(-x)\right] .
\end{aligned}
$$

Then $\operatorname{Dom}(\tilde{T})=\operatorname{Dom}\left(\tilde{T}_{1}\right)$, and $\operatorname{Dom}\left(\tilde{T}_{1}^{*}\right)=\operatorname{Dom}\left(\tilde{T}_{2}^{*}\right)$. According to the hypothesis, $\operatorname{Dom}\left(\left(\tilde{T}_{2}^{*}\right)^{1 / 2}\right)=e^{-z H} H^{1}(\mathbb{R})$, and the fact that $\operatorname{Dom}\left(\tilde{T}^{1 / 2}\right)=e^{-z H} H^{1}(\mathbb{R})$ follows from Lemma 1 and Lemma 2 in [2]. 
Having reduced the problem to the case of the highest-order term, we recall that $\tilde{J}(f, g)=(F \tilde{D} f, \tilde{D} g)=(\tilde{A} f, g)$; formally, $\tilde{A}=\tilde{D}^{*} F \tilde{D}$. Now, consider the form

$$
J(\phi, \psi)=\left(\tilde{F} \frac{d}{d x} \phi, \frac{d}{d x} \psi\right)
$$

on $H^{1}(\mathbb{R})$, where $\tilde{F}=e^{-2 \operatorname{Re}(z) H} F$. Since $\tilde{F} \in L^{\infty}(\mathbb{R})$, and $\operatorname{Re} \tilde{F}(x) \geq \tilde{\rho}>0$, it follows from the Kato conjecture in one dimension that if $A$ is the corresponding $m$-accretive operator (formally $A=(-d / d x) \tilde{F}(d / d x)$ ), then $\operatorname{Dom}\left(A^{1 / 2}\right)=H^{1}(\mathbb{R})$. We want to show that $\operatorname{Dom}\left(\tilde{A}^{1 / 2}\right)=e^{-z H} H^{1}(\mathbb{R})$. We have that

$$
J(\phi, \psi)=\left(\tilde{F} \frac{d}{d x} \phi, \frac{d}{d x} \psi\right)=(A \phi, \psi)
$$

for all $\psi \in \operatorname{Dom}(A) \subseteq H^{1}, \psi \in H^{1}$. On the other hand, for all $\phi, \psi \in H^{1}$,

$$
\begin{aligned}
\tilde{J}\left(e^{-z H} \phi, e^{-z H} \psi\right) & =\left(F \tilde{D} e^{-z H} \phi, \tilde{D} e^{-z H} \psi\right)=\left(F e^{-z H} \frac{d}{d x} \phi, e^{-z H} \frac{d}{d x} \psi\right) \\
& =\left(\tilde{F} \frac{d}{d x} \phi, \frac{d}{d x} \psi\right)=J(\phi, \psi) .
\end{aligned}
$$

Moreover,

$$
J(\phi, \psi)=\left(\tilde{A} e^{-z H} \phi, e^{-z H} \psi\right)=\left(e^{-\bar{z} H} \tilde{A} e^{-z H} \phi, \psi\right),
$$

for all $\phi$ such that $e^{-z H} \phi \in \operatorname{Dom}(\tilde{A}) \subseteq e^{-z H} H^{1}$ and $\psi \in H^{1}$.

Let $S$ denote the operator $e^{i \gamma H} \tilde{A} e^{-i \gamma H}$, with

$$
\operatorname{Dom}(S)=\left\{\phi \in L^{2}(\mathbb{B}) \mid e^{-i \gamma H} \phi \in \operatorname{Dom}(\tilde{A})\right\},
$$

$\gamma \in \mathbb{R}$. Then $S$ is a closed $m$-accretive operator with $\operatorname{Dom}(S) \subseteq H^{1}(\mathbb{R})$, and for all $\phi \in \operatorname{Dom}(S), \psi \in H^{1},(S \phi, \psi)=J(\phi, \psi)$. By uniqueness in the representation theorem for sectorial forms, $S=e^{i \gamma H} \tilde{A} e^{-i \gamma H}=A$, and so $\tilde{A}=e^{-i \gamma H} A e^{i \gamma H}$. Next, the operator $e^{-i \gamma H} A^{1 / 2} e^{i \gamma H}$ with domain $\left\{\phi \in L^{2} \mid e^{i \gamma H} \phi \in \operatorname{Dom}\left(A^{1 / 2}\right)\right\}$ is $m$-accretive, and $\left(e^{-i \gamma H} A^{1 / 2} e^{i \gamma H}\right)^{2}=\tilde{A}$. It follows from uniqueness of the square root of $\tilde{A}$ that $\tilde{A}^{1 / 2}=e^{-i \gamma H} A^{1 / 2} e^{i \gamma H}$, hence $\operatorname{Dom}\left(\tilde{A}^{1 / 2}\right)=e^{-i \gamma H} H^{1}(\mathbb{R})$.

NOTE. The operator $S$ in the proof of Proposition 3 is unitarily equivalent to $\tilde{A}$ when $z=i \gamma$. This is not the case, however, when $z \neq i \gamma$. In order to extend the result in Proposition 3 to all $z \in \mathbb{C}$, we find a wavelet basis for $\operatorname{Dom}(\tilde{A})$ that is a modification of the construction in [1]. 


\section{Wavelet bases}

The wavelet basis used in [1] can be adapted to provide unconditional bases for the domains of the operators considered in Section 2 . This is not entirely surprising, since the growth conditions on the wavelets in [16] are similar to those on the eigenvectors of the operators studied in [4]. The main result, similar to that in [1], involves the operator $\tilde{A}=\tilde{D}^{*} F \tilde{D}$ of the previous section. Recall that $\tilde{D}=e^{-z H}(d / d x) e^{z H}$ so that $\tilde{A}$ depends on $z$.

THEOREM 4. There exists a family of Lipschitz functions $\left\{\tau_{\lambda}(x)\right\}$ belonging to $\operatorname{Dom}(\tilde{A})$ that forms an unconditional basis for each of $L^{2}(\mathbb{R}), e^{-z H} H^{1}(\mathbb{R})$, and $\operatorname{Dom}(\tilde{A})$.

PROOF. Using the notation in [1], let $\Lambda$ denote the collection of all dyadic intervals in $\mathbb{R}$. If $\lambda=\left[k 2^{-j},(k+1) 2^{-j}\right)$, with $k, j \in Z$, then there exists a family of functions $\left\{\theta_{\lambda}(x)\right\}$ of class $C^{2}$ satisfying the following conditions (cf. [16]):

$$
\begin{aligned}
\left|\theta_{\lambda}(x)\right| & \leq C 2^{j / 2} \exp \left(-\gamma\left|2^{j} x-k\right|\right), \\
\left|D \theta_{\lambda}(x)\right| & \leq C 2^{3 j / 2} \exp \left(-\gamma\left|2^{j} x-k\right|\right), \\
\left|D^{2} \theta_{\lambda}(x)\right| & \leq C 2^{5 j / 2} \exp \left(-\gamma\left|2^{j} x-k\right|\right),
\end{aligned}
$$

where $D=-i(d / d x)$ with domain $H^{1}(\mathbb{R})$. In general, $\left\{\theta_{\lambda}(x)\right\}$ is adapted to a function $b(x) \in L^{\infty}(\mathbb{R})$ satisfying $\operatorname{Re} b(x) \geq 1$, in the sense that

$$
\begin{aligned}
\int \theta_{\lambda}(x) b(x) d x & =0, \\
\int x \theta_{\lambda}(x) b(x) d x & =0, \\
\int \theta_{\lambda}(x) \theta_{\mu}(x) b(x) d x & =\delta_{\lambda \mu},
\end{aligned}
$$

where $\lambda, \mu \in \Lambda$. The family $\left\{\theta_{\lambda}(x)\right\}$ forms an unconditional basis for $L^{2}(\mathbb{R})$; that is, for all $f \in L^{2}(\mathbb{R})$, we have $f(x)=\sum \alpha_{\lambda} \theta_{\lambda}(x)$ where $\left\{\alpha_{\lambda}\right\} \in l^{2}(\Lambda)$, and $\|f\|_{2}$ and $\left(\sum\left|\boldsymbol{\alpha}_{\lambda}\right|^{2}\right)^{1 / 2}$ are equivalent norms. Moreover, for all $\lambda \in \Lambda$

$$
\alpha_{\lambda}=\int_{-\infty}^{\infty} f(x) \theta_{\lambda}(x) b(x) d x
$$

We take $b(x)=e^{2 \operatorname{Re}(z) H} F(x)^{-1}$, and following [1], set

$$
\tau_{\lambda}(x)=2^{j} e^{-z H} D^{-1}\left(e^{2 \operatorname{Re}(z) H} F(x)^{-1} \theta_{\lambda}\right)(x),
$$


and

$$
\sigma_{\lambda}(x)=-2^{-j} e^{-z H} D\left(\theta_{\lambda}\right)
$$

(Note that $\tau_{\lambda}$ and $\sigma_{\lambda}$ here are different from those in [1]). It follows from [1] that both $\left\{e^{z H} \tau_{\lambda}\right\}$ and $\left\{e^{z H} \sigma_{\lambda}\right\}$ are unconditional bases for $L^{2}(\mathbb{R})$. It therefore follows that $\left\{\tau_{\lambda}\right\}$ and $\left\{\sigma_{\lambda}\right\}$ are also unconditional bases for $L^{2}(\mathbb{R})$, since each is the image of an unconditional basis under a bounded invertible linear transformation.

To see that $\left\{\tau_{\lambda}\right\}$ is an unconditional basis for $e^{-z H} H^{1}(\mathbb{R})$, recall that in [1] it is shown that $\left\{e^{z H} \tau_{\lambda}\right\}$ is an unconditional basis for $H^{1}(\mathbb{R})$, and that if $g \in H^{1}(\mathbb{R})$, then $g(x)=\sum \beta_{\lambda} e^{2 H} \tau_{\lambda}(x)$, where $\left\{\beta_{\lambda}\right\}$ and $\left\{2^{j} \beta_{\lambda}\right\} \in l^{2}(\Lambda)$, and $\|g\|_{2}$ and $\left(\sum\left|\beta_{\lambda}\right|^{2}\right)^{1 / 2}$ are equivalent norms. Thus, if $f \in e^{-2 H} H^{1}(\mathbb{R})$, then $f(x)=\sum \beta_{\lambda} \tau_{\lambda}(x)$, and $\|f\|_{2}$ and $\left(\sum\left|\beta_{\lambda}\right|^{2}\right)^{1 / 2}$ are also equivalent norms. Conversely, if $\left\{\beta_{\lambda}\right\}$ and $\left\{2^{j} \beta_{\lambda}\right\} \in l^{2}(\Lambda)$, then by [1], $g(x)=\sum \beta_{\lambda} e^{z H} \tau_{\lambda}(x) \in H^{1}(\mathbb{R})$, so that $f=e^{-z H} g=\sum \beta_{\lambda} \tau_{\lambda} \in e^{-z H} H^{1}(\mathbb{R})$.

In order to characterize $\operatorname{Dom}(\tilde{A})$, set $\tilde{D}=e^{-z H} D e^{z H}$. For $f \in \operatorname{Dom}(\tilde{A}) \subseteq$ $e^{-z H} H^{1}(\mathbb{R})$, we have $f=\sum \alpha_{\lambda} \tau_{\lambda}$, where $\left\{\alpha_{\lambda}\right\}$ and $\left\{2^{j} \alpha_{\lambda}\right\} \in l^{2}(\Lambda)$. We then have, for $f \in \operatorname{Dom}(\tilde{A})$ and $g \in e^{-z H} H^{1}(\mathbb{R})$,

$$
(\tilde{A} f, g)=(F \tilde{D} f, \tilde{D} g)=\left(F e^{-z H} D e^{z H}\left(\sum \alpha_{\lambda} \tau_{\lambda}\right), e^{-z H} D e^{z H} g\right) .
$$

Now $D e^{z H} \tau_{\lambda}=2^{j} e^{2 \operatorname{Re}(z) H}\left(F(x)^{-1}\right) \theta_{\lambda}$, and since $\sum \alpha_{\lambda} e^{z H} \tau_{\lambda} \in H^{1}(\mathbb{R})$, we have $D\left(\sum \alpha_{\lambda} e^{z H} \tau_{\lambda}\right)=\sum \alpha_{\lambda} 2^{j} e^{2 \operatorname{Re}(z) H}\left(F(x)^{-1}\right) \theta_{\lambda}$; the term-by-term differentiation is justified because $\left\{2^{j} \alpha_{\lambda}\right\} \in l^{2}(\Lambda)$. Consequently,

$$
\left(F e^{-z H} D e^{z H}\left(\sum \alpha_{\lambda} \tau_{\lambda}\right), e^{-z H} D e^{z H} g\right)=\left(\sum \alpha_{\lambda} 2^{j} \theta_{\lambda}, D e^{z H} g\right) .
$$

Because $f \in \operatorname{Dom}(\tilde{A})$, the latter inner product can be extended to a bounded linear functional on $L^{2}(\mathbb{R})$, and so it follows that $\sum \alpha_{\lambda} 2^{j} \theta_{\lambda} \in H^{1}(\mathbb{R})$. In general, we have that if $f \in H^{1}(\mathbb{R})$, and $f=\sum \beta_{\lambda} \theta_{\lambda}$, then $\left\{\beta_{\lambda} 2^{j}\right\} \in l^{2}(\Lambda)$. Indeed, from (2) and (3) we have

$$
\begin{aligned}
\beta_{\lambda} & =\int_{-\infty}^{\infty} f(x) \theta_{\lambda}(x) b(x) d x=2^{-j} \int_{-\infty}^{\infty} f(x) D\left(e^{z H} \tau_{\lambda}\right)(x) d x \\
& =-2^{-j} \int_{-\infty}^{\infty} D f(x) e^{z H} \tau_{\lambda}(x) d x .
\end{aligned}
$$

Recall that $\left\{e^{z H} \tau_{\lambda}\right\}$ is one of the unconditional bases described in [1], and that those bases satisfy dual estimates (cf. [1,(11)]) that imply $\left\{\int_{-\infty}^{\infty} D f(x) e^{z H} \tau_{\lambda}(x) d x\right\} \in l^{2}(\Lambda)$. Therefore $\left\{\beta_{\lambda} 2^{j}\right\} \in l^{2}(\Lambda)$, and returning to (4), we now have that $\left\{\alpha_{\lambda} 2^{2 j}\right\} \in l^{2}(\Lambda)$, and

$$
\left(\sum \alpha_{\lambda} 2^{j} \theta_{\lambda}, D e^{z H} g\right)=\left(\sum \alpha_{\lambda} 2^{2 j} e^{z H} \sigma_{\lambda}, e^{z H} g\right) .
$$

Conversely, if $\left\{\alpha_{\lambda} 2^{2 j}\right\} \in l^{2}(\Lambda)$, we see from the same proof that $f(x)=\sum \alpha_{\lambda} \tau_{\lambda}(x) \in$ $\operatorname{Dom}(\tilde{A})$. 
An immediate consequence of the proof is the following (cf. [1]):

COROLLARY 1. $\sum \alpha_{\lambda} \tau_{\lambda}$ belongs to $L^{2}(\mathbb{R}), e^{-z H} H^{1}(\mathbb{R})$, or $\operatorname{Dom}(\tilde{A})$ according to whether $\left\{\alpha_{\lambda}\right\}$ belongs to $l^{2}(\Lambda), l^{2}(\Lambda, \omega)$, or $l^{2}\left(\Lambda, \omega^{2}\right)$, respectively, where $\omega=1+2^{2 j}$.

THEOREM 5. $\operatorname{Dom}\left(\tilde{A}^{1 / 2}\right)=e^{-z H} H^{1}(\mathbb{R})$.

PROOF. This follows from Theorem 4, as noted in [1], because of the complex interpolation theorem of J.-L. Lions: if $T$ is a maximal-accretive operator on $\mathscr{H}$ then the $\operatorname{Dom}\left(T^{1 / 2}\right)$ is a complex interpolation space between $\operatorname{Dom}(T)$ and $\mathscr{H}$.

NOTE. As pointed out by the referee, another proof of Theorem 5 is possible, by showing that the operator $\tilde{A}$ is similar to an operator of the form $b D a D$, which in turn is similar to an operator of the form $D a D$ (for which the Kato conjecture is true, by [5] and [1]). Moreover, Theorems 4 and 5 remain true when the Heaviside function is replaced by a more general complex-valued measurable function.

\section{A related example}

The fundamental building blocks $T_{z}$ for the operators considered in Sections 2 and 3 were first defined by Segal [S], and formally correspond to perturbations of $d / d x$ by delta-potentials. While $T_{z}$ is not self-adjoint, the slightly modified operator $T_{i c}=e^{-i c H}(-i d / d x) e^{i c H}$ is, and the determination of $Q\left(T_{i c}\right)$ is an interesting question in its own right. For $c \in \mathbb{R},\left\{T_{i c}\right\}$ is the one-parameter family of self-adjoint extensions of the symmetric operator $-i(d / d x) \mid C_{0}^{\infty}(\mathbb{R} \backslash\{0\})$, which has deficiency indices $(1,1)$. Recall that

$$
Q\left(\frac{1}{i} \frac{d}{d x}\right)=H^{1 / 2}(\mathbb{R})=\left\{\left.\phi \in L^{2}(\mathbb{R})\left|\int\right| k|| \hat{\phi}(k)\right|^{2} d k<\infty\right\} .
$$

Then it is straightforward to show:

PROPOSITION 6. $Q\left(T_{i c}\right)=e^{-i c H} H^{1 / 2}(\mathbb{R})$.

PROOF. This follows immediately from the spectral theorem. If $\int \lambda d E(\lambda)$ is the spectral representation of $D=-i(d / d x)$, then

$$
Q(D)=\left\{\phi \in L^{2}(\mathbb{R})\left|\int\right| k \mid d(E(\lambda) \phi, \phi)<\infty\right\} .
$$


Accordingly, the spectral representation of $T_{i c}$ is $\int \lambda d\left(e^{-i c H} E(\lambda) e^{i c H}\right)$, and so

$$
\begin{aligned}
Q\left(T_{i c}\right) & =\left\{\phi \in L^{2}(\mathbb{R})\left|\int\right| k \mid d\left(e^{-i c H} E(\lambda) e^{i c H} \phi, \phi\right)<\infty\right\} \\
& =\left\{\phi \in L^{2}(\mathbb{R}) \mid e^{i c H} \phi \in Q(D)\right\}=e^{-i c H} H^{1 / 2}(\mathbb{R}) .
\end{aligned}
$$

As an operator, $T_{i c}$ does not preserve the form domain of $D$. However, $T_{i c}$ arises from the sesquilinear form $\left.-i(d / d x) e^{i c H} \phi, e^{i c H} \phi\right)$ on $e^{-i c H} H^{1 / 2}(\mathbb{R})$, and since $\operatorname{Dom}\left(\left|T_{i c}\right|^{1 / 2}\right)=e^{-i c H} H^{1 / 2}(\mathbb{R})$ by Proposition 6, the Kato conjecture is true in this case. If $c$ is not real, there is no corresponding sesquilinear form.

\section{Acknowledgement}

We are grateful to the referee for pointing out the relevance of references [13] and [9] (and that a new proof of the result in [9] may be found in [3]). We wish to thank the referee for making several helpful suggestions which both improved our exposition, and broadened the scope of our paper; see the note following the proof of Theorem 5.

\section{References}

[1] P. Auscher and P. Tchamitchian, 'Ondelettes et conjecture de Kato', C. R. Acad. Sci. Paris Sér I 313 (1991), 63-66.

[2] — , 'Conjecture de Kato sur les ouverts de $\mathbb{R}$ ', Rev. Mat. Iberoamericana 8 (1992), 149-199.

[3] —_- 'Une nouvelle approche de la conjecture de Kato et équations elliptiques complexes en dimension deux', preprint, IRMAR, Université de Rennes I (1993).

[4] P. Chernoff and R. Hughes, 'A new class of point interactions in one dimension', J. Funct. Anal. 111 (1993), 97-117.

[5] R. Coifman, A. McIntosh and Y. Meyer, 'L'intégrale de Cauchy définit un opérateur borné sur $L^{2}(\mathbb{R})$ pour les courbes lipschitziennes', Ann. of Math. 116 (1982), 361-387.

[6] _ - 'The Hilbert transform on Lipschitz curves', Proc. Centre Math. Anal. Aust. Nat. Univ. 1 (1982), 26-69.

[7] E. Fabes, D. Jerison and C. Kenig, 'Multilinear Littlewood-Paley estimates with applications to partial differential equations', Proc. Nat. Acad. Sci. U.S.A 79 (1982), 5746-5750.

[8] T. Kato, Perturbation theory for linear operators (Springer, Berlin, 1966).

[9] C. Kenig and Y. Meyer, 'Kato's square roots of accretive operators and Cauchy kernels on Lipschitz curves are the same', in: Recent progress in Fourier analysis, Math. Studies 111 (North-Holland, New York, 1985) pp. 123-145.

[10] A. McIntosh, 'On the comparability of $A^{1 / 2}$ and $A^{* 1 / 2}$, Proc. Amer. Math. Soc. 32 (1972), 430-434.

[11] _ 'Heinz inequalities and the perturbation of spectral families', Macquarie Mathematics Report, 79-0006, 1979.

[12] — 'The square root problem for elliptic operators', in: Functional analytic methods for partial differential equations, Lecture Notes in Math. 1450 (Springer, Berlin, 1990) pp. 122-140. 
[13] - 'Operators which have an $H^{\infty}$ functional calculus', Centre Math. Anal. Austral. Nat. Univ. AT14 (1986), 210-231.

[14] M. Reed and B. Simon, Methods of modern mathematical physics, II: Fourier analysis, selfadjointness (Academic Press, New York, 1975).

[15] I. Segal, 'Singular perturbations of semigroup generators', in: Linear operators and approximation, Proc. Conf. Oberwohlfach, 1971, Internat. Ser. Numer. Math. 20 (Birkhäuser, Basel, 1972) pp. 54-61.

[16] Ph. Tchamitchian, 'Ondelettes et intégrale de Cauchy sur les courbes lipschitziennes', Ann. of Math. 129 (1989), 641-649.

Department of Mathematics

Bryn Mawr College

Bryn Mawr, PA 19010

USA

e-mail: rhughes@cc.brynmawr.edu
Department of Mathematics University of California, Berkeley Berkeley, CA 94720 USA e-mail: chernoff@math.berkeley.edu 